\title{
Charge Asymmetry of Muons Generated by Laser- Induced Nuclear Processes in Ultra-dense Hydrogen $D(0)$ and $p(0)$
}

Leif Holmlid ( $\nabla$ holmlid@chem.gu.se )

University of Gothenburg

\section{Research Article}

Keywords: ultra-dense hydrogen, laser-induced processes, MeV particles, mesons

Posted Date: November 10th, 2021

DOl: https://doi.org/10.21203/rs.3.rs-493124/v1

License: (c) (1) This work is licensed under a Creative Commons Attribution 4.0 International License.

Read Full License 


\section{Abstract}

Laser-induced nuclear reactions in ultra-dense hydrogen $\mathrm{H}(0)$ (review in Physica Scripta 2019) give mesons (kaons and pions) which decay to muons. The process which gives the mesons is baryon annihilation (Holmlid, J. Hydrogen Energy 2021; Holmlid and Olafsson, High Energy Density Phys. 2021). The sign of the muons detected depends on the initial baryons, with $D(0)$ in the meson source producing mainly positive muons and $\mathrm{p}(0)$ producing mainly negative muons. This charge asymmetry was reported in Holmlid and Olafsson (Heliyon 2019), and has been confirmed by later experiments with a coil current transformer as beam detector, also in another lab (unpublished). The current coil detector would give no signal from the muons if charge symmetry existed. The charge asymmetry of the muons seems first to be at variance with charge conservation. An analysis of the results which includes charge conservation is given here. It agrees with the standard model of particle physics. Using $D(0)$, the asymmetry is, as previously, proposed to be due to capture of $\mu$ - in D atoms and D2 molecules. This gives emission of mainly $\mu+$ and a fraction of $>50 \%$ of $\mu+$ from $D(0)$. In $p(0)$, the capture rate of $\mu$ - is lower than in $D(0)$. The emitted number of $\mu+$ will be decreased by reaction between $\mu+$ and abundant electrons, forming muonium particles. This effect decreases the fraction of emitted $\mu+$ for both $p(0)$ and $D(0)$, and it is proposed to be the main reason for a larger fraction of emitted $\mu$ - in the case of $p(0)$.

\section{Introduction}

The physics of the quantum material ultra-dense hydrogen $\mathrm{H}(0)$ was described in a review paper in Physica Scripta in 2019 [1], and the science and technology of its production were reviewed in 2021 [2]. $\mathrm{H}(0)$ has an experimental interatomic distance of $2.3 \pm 0.1 \mathrm{pm}$ in spin level $s=2,[1,3]$ and $0.56 \mathrm{pm}$ at spin level $s=1$ [4]. This means that $\mathrm{H}(0)$ is the densest form of matter that exists anywhere in the Universe [5,6]. As described previously [1], three different methods have been used to verify the short interatomic distances. It is expected [7] that $\mathrm{H}(0)$ is superfluid and superconductive. This is indeed observed at room temperature [8-10]. Definite transition temperatures exist to a non-superfluid state at a few hundred $\mathrm{K}$ above room temperature [10]. $\mathrm{H}(0)$ has been studied using laser-induced Coulomb explosions (CE) (this is the first method used to verify the pm distances). This method has been coupled to time-of-flight (TOF) and time-of-flight mass spectrometry (TOF-MS) [1,3] detection of the CE fragments. The second method for verification of the pm distances in $\mathrm{H}(0)$ is rotational emission spectroscopy $[1,11,12]$ which gives the inter-atomic distances as a function of the spin quantum number $s$ with a precision in the femtometer range.

The third method used for verifying the pm distances in $\mathrm{H}(0)$ is based on the observations of nuclear processes in $\mathrm{H}(0)$, both spontaneous and laser-induced, which will only be possible at such short distances. The laser-induced nuclear processes eject particles in the $\mathrm{MeV}$ range, from relatively weak laser pulses at $<0.4 \mathrm{~J}[1,13,14] .{ }^{4} \mathrm{He}$ and ${ }^{3} \mathrm{He}$ ions have been observed, which is a clear indication of nuclear fusion processes [15]. Already in 2015, heat generation above break-even was reported with a relatively weak laser [16]. This was the first report on sustained nuclear fusion above break-even and indeed the first working fusion process which was reported with deuterium as fuel. It has later been 
concluded that muon-catalyzed fusion was an important process giving these results. The laser-induced $\mathrm{MeV}$ meson and muon signals are so large that they can be measured directly by a fast oscilloscope connected to a metal collector inside the vacuum chamber. This large signal gives accurate sub-ns-range timing intensity distributions. The fast particles have been observed at distances up to $2 \mathrm{~m}$ in a vacuum $[13,14,17,18]$. The meson and muon velocities correspond to $10-500 \mathrm{MeV} \mathrm{u}^{-1}$. Magnetic deflection studies were used to confirm that many of the particles, which are formed initially by the laser-induction in $\mathrm{H}(0)$, are neutral [19], thus not trivial protons or electrons. The generation of muons from the laser interaction with $\mathrm{H}(0)$ is confirmed by direct measurements of the accurate decay-time for the muons [20]. Other studies $[21,22]$ confirm that the charged and long-lived neutral kaons as well as the charged pions from the laser-induced processes in $\mathrm{H}(0)$ decay with their well-known decay lifetimes [23-25].

Large fluxes of muons are generated by the patented muon generator [26] with application in muoncatalyzed fusion reactors [27]. Since only negative muons can be used for muon-catalyzed fusion [28], the conditions for generating muons with different signs are of great practical importance and they are studied mainly for this reason. This is a problem for the presently ongoing development of muoncatalyzed fusion, which is the only sustainable nuclear fusion method since it can use deuterium as a fuel [2]. Thus, muon-catalyzed fusion does not require risky handling of radioactive tritium fuel as all other fusion methods under development do [29]. The recent development of annihilation energy generation [22] which can use ordinary hydrogen as fuel, means of course that tritium luckily is no longer needed for any type of energy generation.

\section{Theory For Ultra-dense Hydrogen $\mathrm{H}(0)$}

Ultra-dense hydrogen $\mathrm{H}(0)$ is a quantum material. The fundamental physics of it was recently reviewed [1], thus only the most important properties for the present study are summarized here. The ultra-dense states are characterized by their (mixed) spin quantum number $s=1,2,3 \ldots .$. [1]. The state $s=$ 2 which is formed most easily in the laboratory at low temperature and pressure, has an $\mathrm{H}$-H distance of $2.3 \mathrm{pm}$. The state $s=1$ is formed from $s=2$ both spontaneously at a low rate [30] and by laser pulse induction [31]. In this level $s=1$ at an $\mathrm{H}-\mathrm{H}$ distance of only $0.56 \mathrm{pm}$ [1], it is likely that nuclear reaction takes place rapidly. At similar inter-nuclear distances in muon-catalyzed fusion, the rate constant for fusion $D+D$ is $10^{9} \mathrm{~s}^{-1}[28]$. This means that the laser field is only required for transferring $H(0)$ from $s=2$ to $s=1$ at $0.56 \mathrm{pm}$ distance to initiate the nuclear processes, not to in any way force or drive the nuclear processes.

\section{The Nuclear Reaction Process}

The reason why the nuclear processes described here can take place at all is of course the very short distance between the nucleons, at $0.56 \mathrm{pm}$ in spin state $s=1$ for $\mathrm{H}(0)$ [1]. From this level $s=1$ [4] at an $\mathrm{H}$ $\mathrm{H}$ distance of only $0.56 \mathrm{pm}$ [1], it is likely that nuclear reaction takes place rapidly. At similar inter-nuclear distances in muon-catalyzed fusion, the rate constant for $D+D$ fusion is $10^{9} \mathrm{~s}^{-1}$ [28]. 
The muons formed by meson decay have kinetic energies of $>100 \mathrm{MeV} \mathrm{u}^{-1}$ [17-19]. The meson formation process was identified in [12] as being baryon annihilation. The entire process starts with a beta capture step. The proton undergoing beta capture may be called a quasi-neutron and it is formed in the ultradense state $\mathrm{H}(0)(s=1)$ by the interaction of a proton with an electron with $I=0$ and spin $1 / 2$ from $\mathrm{H}(0)$ spin state $s=2$. Spin may be conserved by a simultaneous spin transfer process in $\mathrm{H}(0) s=2 \rightarrow s=1$ (from $S=\hbar$ to $S=\hbar / 2$ ). This makes the transition spin allowed from an electron + a proton, both with spin $1 / 2$ and spins up and down, to a neutron with spin $1 / 2$. A neutrino with spin $1 / 2$ is also conventionally formed in this process, which gives further freedom for the spin conservation. Since the quasi-neutrons have zero charge, they may undergo oscillations similar to neutrino or neutral kaon oscillations to form their own antiparticles. The recently reported case with oscillations of $D^{0}$ mesons between their particle and antiparticle forms is a close analogue [32]. The process of forming the antiparticles, antiprotons and antineutrons, required for the annihilation reactions will not be dealt with here in detail since it belongs to the field of theoretical particle physics and will be described elsewhere (however see more details in the supplementary file named Appendix). In short, beta capture in a proton can form a neutron or an antineutron, which can then form an antiproton by further beta capture. So the description here continues with the proof of annihilation reactions. Almost all of these processes conserve both lepton and baryon numbers and agree with the standard model of particle physics. The mesons have been identified from their decay time constants [1,12], decaying in well-known reactions [23-25] to muons and sometimes other particles like neutrinos and gamma photons. See further Table 1.

\subsection{Baryon annihilation}

The kaons formed have kinetic energies close to $100 \mathrm{MeV}$ as measured from their dilated decays, see Table 1 and Ref. [12]. It is clear that no other process than annihilation can give such large kaon energies. Two baryons annihilate to form six light mesons (kaons and pions, see further below), [23-25]. From the experiments [12], it is clear that charged pions can be formed initially with both low and high kinetic energy, with one example in Fig, 1 and further results in Table 1. It seems that the kaons are always fast initially but the long-lived neutral kaons can be thermalized. The annihilation reactions of interest here are both proton + antiproton and neutron + antineutron:

$$
\begin{aligned}
& p+\bar{p} \rightarrow 2 \mathrm{~K}^{ \pm}(96 \mathrm{MeV})+2 \pi^{ \pm}(69 \mathrm{MeV})+2 \pi^{ \pm}(0 \mathrm{MeV}) \\
& 2 \times 938.3-2 \times(493.7+96)-2 \times(139.6+69)-2 \times(139.6)= \\
& 1876.6-1875.8=0.8 \mathrm{MeV} \\
& n+\bar{n} \rightarrow 2 \mathrm{~K}^{0}(96 \mathrm{MeV})+2 \pi^{ \pm}(69 \mathrm{MeV})+2 \pi^{ \pm}(0 \mathrm{MeV}) \\
& 2 \times 939.6-2 \times(497.7+96)-2 \times(139.6+69)-2 \times(139.6)= \\
& 1879.2-1883.8=-4.6 \mathrm{MeV}
\end{aligned}
$$

where the kinetic energies within parantheses are taken from Table 1. Since the neutrons have slightly higher energies than the protons, it is assumed that the slightly heavier neutral kaons are formed more easily by neutron annihilation. Neutral pions with mass of $135 \mathrm{MeV}$ may be formed instead of charged 
pions. The kinetic energy of such pions has not been measured since they decay much too fast to give any measurable decay dilation.

The annihilation energy cycles above are almost exactly closed, for neutrons with a precision of $2.4 \times 10^{-3}$ and for protons with a precision of $4 \times 10^{-5}$. Of course, this very high precision is somewhat accidental since the spreads in kinetic energies giving the error limits in Table 1 are not included in the energy cycles above. These error limits give a further uncertainty of $\pm 2-4 \mathrm{MeV}$ in the energy cycles, which is still quite negligible. These results support the annihilation interpretation, with no other process possible to give the observed high particle energies anyway.

\subsection{Kaon decay}

The decays of kaons and pions are well known [23-25]. They often give muons by processes similar to pair production, or give muons with the same sign as the initially formed decaying charged kaon. Charge is conserved so an equal number of positive and negative muons is expected to be formed. In some decays for both neutral and charged kaons, neutral pions are formed instead of charged pions. The neutral pions do not decay to muons but instead each neutral pion decays to two high-energy gammas. These processes thus decrease the number of muons formed.

\subsection{Formation and detection of muonium}

Most mesons decay close to the meson generator, thus most muons are formed there. The muons have initial kinetic energies of 100-500 MeV and are thus close to their ionization minimum [33], but they can be slowed down rapidly enough, so that the decay times observed can be close to the values at rest [20]. The hydrogen gas pressure at the generator is of the order of mbars and the muons will lose energy both by gas collisions and by collisions in the closely located metal parts in the apparatus. After the muons have reached a low kinetic energy, the possibility of recombination of positive muons with the abundant electrons in the cooled plasma region increases, giving muonium $\mu^{+} e$. Such particles are not detectable by wire coils on ferrite toroids, which are used for detecting beams and pulses of charged particles [34] and which have been used in our experiments. It is also likely that muonium particles can pass undetected through the type of Al foil collector used in our experiments [17-19], since they are small and neutral. This process decreases the number of bare positive muons in the beam and it will thus give a larger fraction of negative muons in the beam.

\section{Conclusions}

The observed charge asymmetry in the muon emission from the baryon annihilation reactions in the muon source is due to secondary effects like muon capture and muonium formation. These conclusions will hopefully enable further efficient development of intense muon sources with a high fraction of negative muons needed for the only known sustainable fusion process namely muon-catalyzed fusion. 


\section{References}

[1] L. Holmlid and S. Zeiner-Gundersen, Ultradense protium $\mathrm{p}(0)$ and deuterium $\mathrm{D}(0)$ and their relation to ordinary Rydberg matter: a review. http://doi.org/10.1088/1402-4896/ab1276. Physica Scripta 74 (7) 2019.

[2] L. Holmlid, A. Kotarba and P. Stelmachowski, "Production of ultra-dense hydrogen $\mathrm{H}(0)$ : a novel nuclear fuel". International Journal of Hydrogen Energy, https://doi.org/10.1016/j.ijhydene.2021.02.221

[3] L. Holmlid, Excitation levels in ultra-dense hydrogen $\mathrm{p}(-1)$ and $\mathrm{d}(-1)$ clusters: structure of spin-based Rydberg Matter. Int. J. Mass Spectrom. 352 (2013) 1- 8. DOI: 10.1016/j.ijms.2013.08.003.

[4] L. Holmlid, "Excitation levels in ultra-dense hydrogen $\mathrm{p}(-1)$ and d(-1) clusters: structure of spin-based Rydberg Matter". Int. J. Mass Spectrom. 352 (2013) 1- 8. DOI: 10.1016/j.ijms.2013.08.003.

[5] L. Holmlid, " Ultra-dense hydrogen $\mathrm{H}(0)$ as stable dark matter in the Universe: extended red emission spectra agree with rotational transitions in $\mathrm{H}(0)$." The Astrophysical Journal, 866:107 (4pp), https://doi.org/10.3847/1538-4357/aadda1

[6] L. Holmlid, "Ultra-dense hydrogen $\mathrm{H}(0)$ as dark matter in the universe: new possibilities for the cosmological red-shift and the cosmic microwave background radiation”. Astrophys. Space Sci. (2019) 364: 141. DOI: 10.1007/s10509-019-3632-y.

[7] E. Babaev, A. Sudbø, and N. W. Ashcroft, A superconductor to superfluid phase transition in liquid metallic hydrogen. Nature 431, 666 (2004).

[8] P. U. Andersson and L. Holmlid, Superfluid ultra-dense deuterium D(-1) at room temperature". Phys. Lett. A 375, 1344 (2011). doi:10.1016/j.physleta.2011.01.035.

[9] P. U. Andersson, L. Holmlid, and S. R. Fuelling, Search for superconductivity in ultra-dense deuterium $\mathrm{D}(-1)$ at room temperature: depletion of D(-1) at field strength > 0.05 T. Supercond. Novel Magn. 25 (2012) 873-882. DOI: 10.1007/s10948-011-1371-6.

[10] L. Holmlid and B. Kotzias, Phase transition temperatures of $405-725 \mathrm{~K}$ in superfluid ultra-dense hydrogen clusters on metal surfaces. AIP Advances 6, 045111 (2016). doi: 10.1063/1.4947276.

[11]. L. Holmlid, Emission spectroscopy of IR laser-induced processes in ultra-dense deuterium $D(0)$ : Rotational transitions with spin values $s=2,3$ and 4. Mol. Struct. 1130 (2017) 829-836. Doi: 10.1016/j.molstruc.2016.10.091.

[12]. L. Holmlid, Rotational emission spectroscopy in ultra-dense hydrogen $p(0)$ and $p_{x} D_{y}(0)$ : groups $p_{N}$, $\mathrm{pD}_{2}, \mathrm{p}_{2} \mathrm{D}$ and $(\mathrm{pD})_{\mathrm{N}}$. Journal of Molecular Structure 1173, 567-573 (2018) https://doi.org/10.1016/j.molstruc.2018.06.116. 
[13] L. Holmlid, Two-collector timing of 3-14 MeV/u particles from laser-induced processes in ultra-dense deuterium. Int. J. Modern Phys. E 22 (2013) 1350089. DOI: 10.1142/S0218301313500894

[14] F. Olofson and L. Holmlid, Electron-positron pair production observed from laser-induced processes in ultra-dense deuterium D(-1). Laser Part. Beams 32 (2014) 537-548. doi:10.1017/S0263034614000494.

[15] F. Olofson and L. Holmlid, Time-of-flight of He ions from laser-induced processes in ultra-dense deuterium D(0). Int. J. Mass Spectrom. 374 (2014) 33-38. doi: 10.1016/j.ijms.2014.10.004.

[16] L. Holmlid, Heat generation above break-even from laser-induced fusion in ultra-dense deuterium. AIP Advances 5 (2015) 087129. doi: 10.1063/1.4928572.

[17] L. Holmlid, MeV particles in a decay chain process from laser-induced processes in ultra-dense deuterium D(0),. Int. J. Modern Phys. E 24, 1550026 (2015). DOI: 10.1142/S0218301315500263.

[18] L. Holmlid, "Nuclear particle decay in a multi-MeV beam ejected by pulsed-laser impact on ultra-dense hydrogen H(0)", Int. J. Modern Phys. E 24, 1550080 (2015). DOI: 10.1142/S0218301315500809.

[19]. Holmlid, "Mesons from laser-induced processes in ultra-dense hydrogen H(0)".PLOS ONE 12 (2017) e0169895. doi:10.1371/journal.Pone.0169895. Correct file at https://gup.ub.gu.se/file/208005.

[20]. L. Holmlid and S. Olafsson, Decay of muons generated by laser-induced processes in ultra-dense hydrogen. Heliyon 5 (6) (2019) e01864. Doi: 10.1016/j.heliyon.2019.e01864.

[21] L. Holmlid, Laser-induced nuclear processes in ultra-dense hydrogen take place in small nonsuperfluid $\mathrm{H}_{N}(0)$ clusters. Journal of Cluster Science, 30(1), (2019) 235-242. DOI: 10.1007/s10876-0181480-5.

[22] L. Holmlid,"Energy production by laser-induced annihilation in ultradense hydrogen $\mathrm{H}(0)$ ". J. Hydrogen Energy (2021) https://doi.org/10.1016/j.ijhydene.2021.01.212.

[23] C. Nordling and J. Österman, Physics Handbook (Studentlitteratur, Lund, Sweden 1988).

[24] W. E. Burcham and M. Jobes, "Nuclear and Particle Physics", Pearson, Harlow 1995.

[25] Particle Data Group, M. Tanabashi et al. (Particle Data Group), Phys. Rev. D 98, 030001 (2018). (URL: http://pdg.lbl.gov).

[26] . Holmlid, "Apparatus for generating muons with intended use in a fusion reactor". Patent nr SE 539684 C 2.

[27] L. Holmlid, Existing source for muon-catalyzed nuclear fusion can give MW thermal fusion generator. Fusion Science and Technology, 75:3, 208-217, DOI: 10.1080/15361055.2018.1546090. 
[28] Balin DV, Ganzha VA, Kozlov SM, Maev EM, Petrov GE, Soroka MA, Schapkin GN, Semenchuk GG, Trofimov VA, Vasiliev AA, Vorobyov AA, Voropaev NI, Petitjean C, Gartner B, Lauss B, Marton J, Zmeskalc J, Case T, Crowe KM, Kammel P, Hartmann FJ, Faifman MP. High precision study of muon catalyzed fusion in $D_{2}$ and $H D$ gases, Phys. Part. Nuclei 42, 185-214 (2011). https://doi.org/10.1134/S106377961102002X.

[29] L.Holmlid, "Muon-catalyzed fusion and annihilation energy generation will supersede nonsustainable T+D nuclear fusion”. DOI: 10.21203/rs.3.rs-148681/v1.

[30] L. Holmlid and S. Olafsson, Spontaneous ejection of high-energy particles from ultra-dense deuterium D(0), Int. J. Hydr. Energy 40, 10559-10567 (2015). DOI: 10.1016/j.ijhydene.2015.06.116.

[31] L. Holmlid and S. Olafsson, Charged particle energy spectra from laser-induced processes: nuclear fusion in ultra-dense deuterium D(0), Int. J. Hydrogen Energy 41, 1080-1088 (2016). doi:10.1016/j.ijhydene.2015.10.072.

[32]LHCb collaboration (several hundred names). CERN, Observation of the mass difference between neutral charm-meson eigenstates, arXiv:2106.03744v1 [hep-ex] 7 Jun 2021.

[33]. D.E. Groom, N.V. Mokhov, S. Striganov, Muon stopping power and range tables $10 \mathrm{MeV}-100 \mathrm{TeV}$, Atomic Data Nucl. Data Tables 76 (2) (2001) (LBNL-44742).

[34]. https://uspas.fnal.gov/materials/09UNM/BeamCurrentMonitors.pdf. 2009. Read 21-05-03.

[35] D.F. Measday, The nuclear physics of muon capture, Phys. Rep. 354 (2001) 243. doi: 10.1016/S03701573(01)00012-6.

[36] Berezhiani, Z. Neutron lifetime puzzle and neutron-mirror neutron oscillation . Eur. Phys. J. C79, 484 (2019). https://doi.org/10.1140/epjc/s10052-019-6995-x

[37] R. N. Mohapatra, Neutron-Anti-Neutron Oscillation: Theory and Phenomenology, Phys. G: Nucl. Part. Phys.36(2009) 104006, doi: 10.1088/0954-3899/36/10/104006.

[38] 2020 Review of Particle Physics. Limit on $\mathrm{n} \mathrm{n}^{-}$oscillations. P.A. Zyla et al. (Particle Data Group), Prog. Theor. Exp. Phys. 2020, $083 C 01$ (2020)

[39] L. Holmlid, "Laser-induced nuclear processes in ultra-dense hydrogen take place in small nonsuperfluid $\mathrm{H}_{\mathrm{N}}(0)$ clusters". Journal of Cluster Science, 30(1), (2019) 235-242. DOI: 10.1007/s10876-0181480-5.

[40] L. Holmlid and S. Zeiner-Gundersen, "Future interstellar rockets may use laser-induced annihilation reactions for relativistic drive". Acta Astronautica 175 (2020) 32-36. https://doi.org/10.1016/j.actaastro.2020.05.034. 
[41] L. Holmlid,"Nuclear particle decay in a multi-MeV beam ejected by pulsed-laser impact on ultra-dense hydrogen H(0)". Int. J. Modern Phys. E 24 (2015) 1550080. DOI: 10.1142/S0218301315500809

[42] L. Holmlid, "Leptons from decay of mesons in the laser-induced particle pulse from ultra-dense protium p(0)”. Int. J. Modern Phys. E 25 (2016) 1650085. DOI: 10.1142/S0218301316500853.

\section{Tables}

Table I. Summary of meson decay-time results [22]. The decay-time error limits given are standard errors

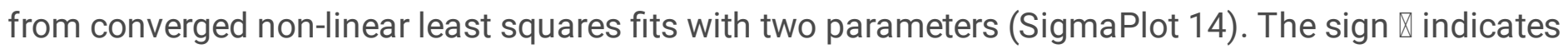
manual adjustments of the rate parameters to fit the experimental curves visually, as described in detail in the relevant references, with one example in Fig. 1.

\begin{tabular}{|c|c|c|c|c|c|c|}
\hline $\begin{array}{l}\text { Observed } \\
\text { decay-time } \\
\text { (ns) }\end{array}$ & Particle & $\begin{array}{l}\text { Particle } \\
\text { decay-time at rest } \\
\text { (ns) [25] }\end{array}$ & $\begin{array}{l}\text { Dilation } \\
\text { factor }\end{array}$ & Velocity/c & $\begin{array}{l}\text { Kinetic } \\
\text { energy } \\
(\mathrm{MeV})\end{array}$ & $\begin{array}{l}\text { Fig. No. or } \\
\text { Refs. [] }\end{array}$ \\
\hline$<2$ & & 0.09 & - & - & - & Fig.1. \\
\hline$\otimes 13$ & $\mathrm{~K}^{ \pm}$ & 12.38 & 1.05 & 0.305 & 24.4 & {$[1,27,39,40]$} \\
\hline $\begin{array}{l}14.81 \pm \\
0.05\end{array}$ & $\mathrm{~K}^{ \pm}$ & 12.38 & 1.196 & 0.549 & $95.7 \pm 2.0$ & \\
\hline$\otimes 26$ & $\pi^{ \pm}$ & 26.033 & 1.0 & low & low & {$[1,27,39]$} \\
\hline $25.92 \pm 0.04$ & $\pi^{ \pm}$ & 26.033 & 1.0 & low & low & \\
\hline $\mathbb{8} 39$ & $\pi^{ \pm}$ & 26.033 & 1.5 & 0.745 & 68.9 & $\begin{array}{l}\text { Fig. } 1 . \\
{[41,42]}\end{array}$ \\
\hline $40.43 \pm 0.10$ & $\pi^{ \pm}$ & 26.033 & 1.555 & 0.766 & $\begin{array}{l}76.7 \pm \\
0.5\end{array}$ & \\
\hline$\otimes 52$ & & 51.16 & 1.016 & 0.18 & 8 & {$[1,20,40]$} \\
\hline $61.3 \pm 0.07$ & & 51.16 & 1.198 & 0.551 & $\begin{array}{l}97.4 \pm \\
0.7\end{array}$ & \\
\hline
\end{tabular}

\section{Figures}




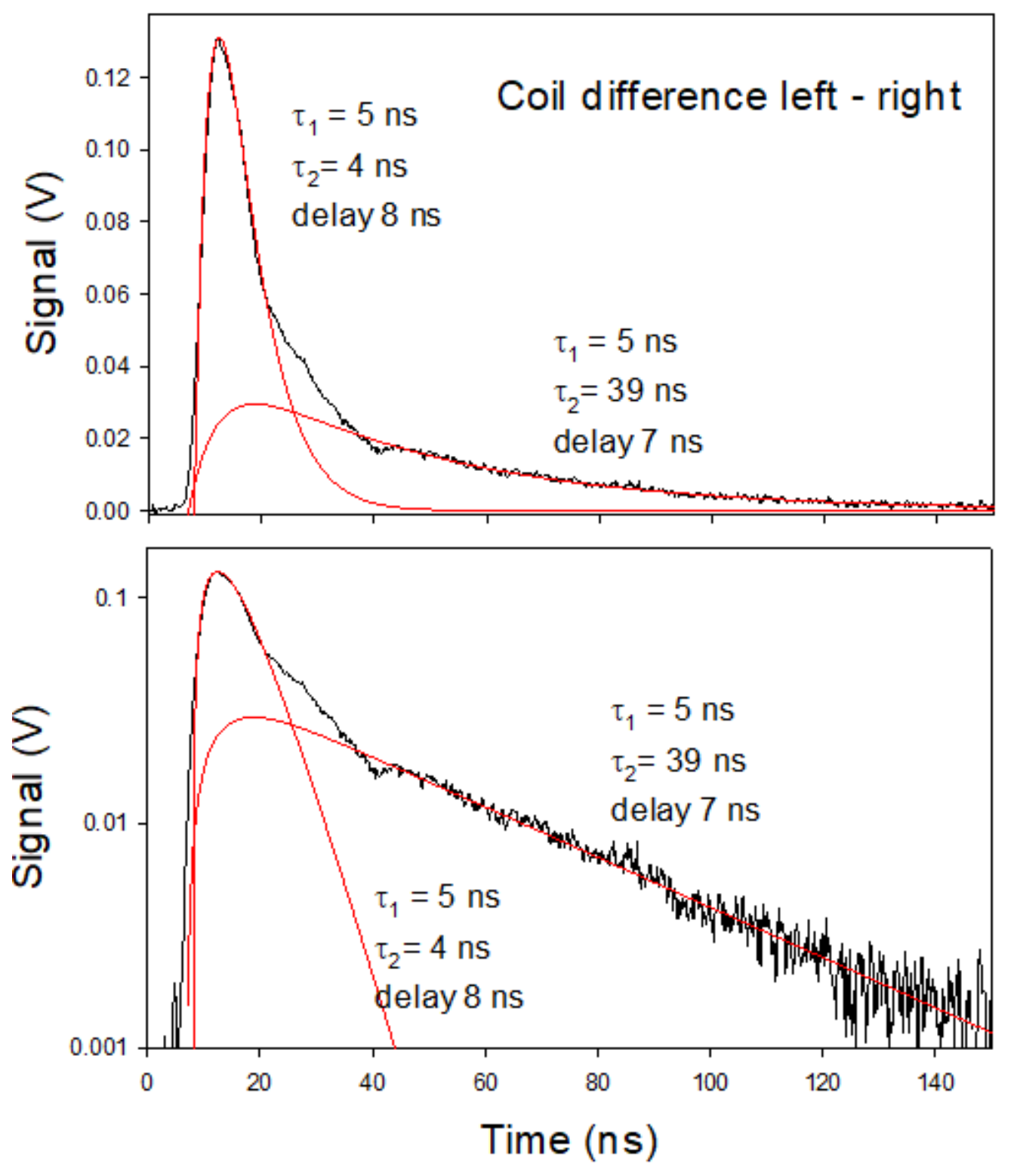

\section{Figure 1}

Linear and logarithmic plots of the same data, the differential signal from a current coil with the mainly positive muon signal passing through its center. $0.4 \mathrm{~J}, 10 \mathrm{~Hz}$ pulse repetition, $1064 \mathrm{~nm}$ laser light. $\mathrm{D}(0)$ in the meson source in a vertical chamber, 0.2 mbar D2. Current coil with 19 turns of wire on a ferrite toroid, at $58 \mathrm{~cm}$ from laser target. The first peak follows the laser peak shape and has thus a time constant $<2$ ns. It is proposed to be muons from the decay of short-lived neutral kaons. See Table 1. The 39 ns time constant is muons from time dilated pion decay as indicated in Table 1, with a kinetic energy of the pions of close to $70 \mathrm{MeV}$.

\section{Supplementary Files}

This is a list of supplementary files associated with this preprint. Click to download. 
- Appendix.doc

Page 11/11 